\title{
DIFFERENCE SEQUENCE SPACES
}

\author{
A.K. GAUR \\ Department of Mathematics \\ Duquesne University \\ Pittsburgh, PA 15282, U.S.A. \\ and \\ MURSALEEN* \\ Department of Mathematics \\ Aligarh Muslim University \\ Aligarh 202002, INDIA
}

(Received April 17, 1996 and in revised form July 29, 1996)

ABSTRACT. In [1]

$$
S_{r}(\Delta):=\left\{x=\left(x_{k}\right):\left(k^{r}\left|\Delta x_{k}\right|\right)_{k=1}^{\infty} \in c_{0}\right\}
$$

for $r \geq 1$ is studied. In this paper, we generalize this space to $S_{r}(p, \Delta)$ for a sequence of strictly positive reals. We give a characterization of the matrix classes $\left(S_{r}(p, \Delta), \ell_{\infty}\right)$ and $\left(S_{r}(p, \Delta), \ell_{1}\right)$.

KEY WORDS AND PHRASES: Difference sequence spaces, Köthe-Toeplitz duals, matrix transformations.

1991 AMS SUBJECT CLASSIFICATION CODES: 40H05, 46A45.

\section{INTRODUCTION}

Let $\ell_{\infty}, c$ and $c_{0}$ be the sets of all bounded, convergent and null sequences of $x=\left(x_{k}\right)_{1}^{\infty}$, respectively. Let $w$ denote the set of all complex sequences and $\ell_{1}$ denote the set of all convergent and absolutely convergent series.

Let $z$ be any sequence and $Y$ be any subset of $w$. Then

$$
z^{-1} \cdot Y=\left\{x \in w: z x=\left(z_{k} x_{k}\right)_{1}^{\infty} \in Y\right\}
$$

For any subset $X$ of $w$, the sets

$$
X^{\alpha}=\bigcap_{x \in X}\left(x^{-1} \cdot \ell_{1}\right) \text { and } X^{\beta}=\bigcap_{x \in X}\left(x^{-1} \cdot c s\right)
$$

are called the $\alpha$ - and $\beta$-duals of $X$.

We define the linear operators $\Delta, \Delta^{-1}: w \rightarrow w$ by

$$
\Delta x=\left(\Delta x_{k}\right)_{1}^{\infty}=\left(x_{k}-x_{k+1}\right)_{1}^{\infty},
$$

and

$$
\Delta^{-1} x=\left(\Delta^{-1} x_{k}\right)_{1}^{\infty}=\left(\sum_{\jmath=1}^{k-1} x_{\jmath}\right)_{1}^{\infty},
$$

such that 


$$
\Delta^{-1} x_{1}=0
$$

Let

$$
S_{r}(\Delta):=\left\{x \in w:\left(k^{r}\left|\Delta x_{k}\right|\right)_{k=1}^{\infty} \in c_{0}\right\} \text {, see [1] }
$$

In this paper we extend the space $S_{r}(\Delta)$ to $S_{r}(p, \Delta)$ in the same manner as $c_{0}, c, \ell_{\infty}$ were extended to $c_{0}(p), c(p), \ell_{\infty}(p)$, respectively (cf. [2],[3],[4]). We also determine the $\alpha$ - and $\beta$-duals of our new sequence space. Let $p=\left(p_{k}\right)_{1}^{\infty}$ be an arbitrary sequence of positive reals and $r \geq 1$, then we define

$$
S_{r}(p, \Delta):=\left\{x \in w:\left(k^{r} \Delta x_{k}\right)_{1}^{\infty} \in c_{0}(p)\right\},
$$

where

$$
c_{0}(p):=\left\{x \in w: \lim _{k \rightarrow \infty}\left|x_{k}\right|^{p_{k}}=0\right\} .
$$

If $p=e=(1,1,1, \ldots)$, then the set $S_{r}(p, \Delta)$ reduces to the set $S_{r}(\Delta)$. For $r=0, S_{r}(p, \Delta)$ is the same as $\Delta c_{0}(p)$ (cf. [5],[6],[7]).

We will need the following lemmas:

LEMMA 1 (Corollary 1 in [7]). Let $\left(P_{n}\right)_{n=1}^{\infty}$ be a sequence of nondecreasing positive reals. Then ,$a \in\left(P_{n}\right)^{-1} \cdot c s$ implies $R=\left(R_{n}\right) \in\left(P_{n}\right)^{-1} \cdot c_{0}$ where $R_{n}=\sum_{k=n+1}^{\infty} a_{k}(n=1,2, \ldots)$.

LEMMA 2 (Lemma 1 (b) in [8]). Let $p=\left(p_{k}\right)_{k=1}^{\infty}$ be a strictly positive sequence such that $p \in \ell_{\infty}$ Then $A \in\left(c_{0}(p), \ell_{1}\right)$ if and only if

$$
B(M)=\sup _{\substack{N \in \mathbb{N} \\ N \text { finite }}}\left(\sum_{k=1}^{\infty}\left|\sum_{n \in N} a_{n k}\right| M^{-1 / p_{k}}\right)<\infty
$$

for some integer $M \geq 2$.

\section{THE $\alpha$-AND $\beta$-DUALS OF $S_{r}(p, \Delta)$}

THEOREM 2.1. Let $p=\left(p_{k}\right)_{1}^{\infty}$ be a strictly positive sequence and $r \geq 1$. Then

$$
\left[S_{r}(p, \Delta)\right]^{\alpha}=\bigcup_{N>1} D_{r}^{(1)}(p)
$$

$$
\left[S_{r}(p, \Delta)\right]^{\beta}=C_{r}(p)=\bigcap_{v \in c_{0}^{+}} D_{r}^{(2)}(p) \bigcap \bigcup_{N>1} D_{r}^{(3)}(p)
$$

where

$$
\begin{aligned}
D_{r}^{(1)}(p): & =\left(\Delta_{r}^{-1} N^{-1 / p}\right)^{-1} \cdot \ell_{1}=\left\{a \in w: \sum_{k=1}^{\infty}\left|a_{k}\right|\left|\sum_{j=1}^{k-1} \frac{N^{-1 / p_{j}}}{j^{r}}\right|<\infty\right\} \\
D_{r}^{(2)}(p): & =\left(\Delta_{r}^{-1} v^{1 / p}\right)^{-1} \cdot c s=\left\{a \in w: \sum_{k=1}^{\infty} a_{k} \sum_{j=1}^{k-1} \frac{v_{j}^{1 / p_{j}}}{j^{r}} \text { converges }\right\}, \\
D_{r}^{(3)}(p): & =\left\{a \in w: R \in\left(\frac{N^{-1 / p}}{k^{r}}\right)^{-1} \cdot \ell_{1}\right\}=\left\{a \in w: \sum_{k=1}^{\infty}\left|R_{k}\right| \frac{N^{-1 / p_{k}}}{k^{r}}<\infty\right\} \\
\Delta_{r} x & =\left(k^{r} \Delta x_{k}\right)_{k=1}^{\infty}, \Delta_{r}^{-1} x=\left(k^{r} \Delta^{-1} x_{k}\right)_{k=1}^{\infty},
\end{aligned}
$$

and $c_{0}^{+}$is the set of all positive sequences in $c_{0}$.

PROOF. (a) Let $a \in \bigcup_{N>1} D_{r}^{(1)}(p)$. Then

$$
a \cdot s\left(1 / N_{0}\right) \in \ell_{1} \quad \text { for some } \quad N_{0} \geq 2,
$$


where

$$
s\left(1 / N_{0}\right)=\left(s_{k}\left(\frac{1}{N_{0}}\right)\right)_{k=1}^{\infty}=\left(\sum_{j=1}^{k-1} \frac{N_{0}^{-1 / p_{j}}}{j^{r}}\right)_{k=1}^{\infty} .
$$

Since $s\left(\frac{1}{N_{0}}\right)$ is increasing, (2.1) implies that

$$
a \in \ell_{1} \text {. }
$$

Let $x \in S_{r}(p, \Delta)$. Then for a given $N_{0} \in \mathbb{N}$, there exists an $M=M\left(N_{0}\right) \in \mathbb{N}$ such that $\sup _{k \geq M}\left|k^{r} \Delta x_{k}\right|^{p_{k}}<\frac{1}{N_{0}}$, and hence $\left|\Delta x_{k}\right| \leq \frac{N_{0}^{-1 / p_{k}}}{k^{r}}$ for all $k=1,2, \ldots$, and consequently by (2.1) we have

$$
\sum_{k=1}^{\infty}\left|a_{k}\right| \sum_{\jmath=1}^{k-1}\left|\Delta x_{\jmath}\right| \leq \sum_{k=1}^{\infty}\left|a_{k}\right| s_{k}\left(1 / N_{0}\right)<\infty
$$

Finally, by (2.2) and (2.3), we get

$$
a \in\left[S_{r}(p, \Delta)\right]^{\alpha} .
$$

Let $a \notin \bigcup_{N>1} D_{r}^{(1)}(p) \quad$ Then we can determine a strictly increasing sequence $(k(m))_{m=1}^{\infty}$ of integers such that $k(1)=1$ and

$$
\sum_{k=k(m)}^{k(m+1)-1}\left|a_{k}\right| s_{k}(1 /(m+1))>1 \quad(m=1,2, \ldots) .
$$

We define the sequence $x=\left(x_{k}\right)$ by

$$
x_{k}=\sum_{i=1}^{m} \sum_{J=(k(2))}^{\min \{k-1, k(i+1)-1\}} \frac{(i+1)^{-1 / p}}{j^{r}}, \quad(k(m) \leq k \leq k(m+1)-1 ; m=1,2, \ldots) .
$$

Then $x \in S_{r}(p, \Delta)$ and

$$
\sum_{k=1}^{\infty}\left|a_{k}\right|\left|x_{k}\right|=\sum_{m=1}^{\infty} \sum_{k=k(m)}^{k(m+1)-1}\left|a_{k} x_{k}\right|>\infty
$$

which proves that

$$
a \notin\left[S_{r}(p, \Delta)\right]^{\alpha}
$$

Hence, $\left[S_{r}(p, \Delta)\right]^{\alpha}=\bigcup_{n>1} D_{r}^{(1)}(p)$.

(b) Let $a \in C_{r}(p)$. Then $a \in c s$, and Abel's summation by parts yields

$$
\sum_{k=1}^{n} a_{k} x_{k}=-\sum_{k=1}^{n-1} R_{k} \Delta x_{k}+R_{n} \sum_{k=1}^{n-1} \Delta x_{k}+x_{1} \sum_{k=1}^{n} a_{k} \text { for all } x, \quad(n=1,2, \ldots) .
$$

Further

$$
R \in\left(\frac{N_{0}^{-1 / p}}{k^{r}}\right) \cdot \ell_{1} \text { for some integer } N_{0} \geq 2 .
$$

Let $x \in S_{r}(p, \Delta)$. Then there is a sequence $v \in c_{0}^{+}$such that

$$
\left|\Delta x_{k}\right| \leq \frac{v_{k}^{1 / p_{k}}}{k^{r}}(k=1,2, \ldots) \text { and }\left|\Delta x_{k}\right| \leq \frac{N_{0}^{-1 / p_{k}}}{k^{r}}
$$

for all sufficiently large $k$. Now, by (2.5) 


$$
\sum_{k=1}^{\infty}\left|R_{k}\right|\left|\Delta x_{k}\right|<\infty
$$

Hence

$$
R \Delta x \in \ell_{1} \subset c s .
$$

Finally, by Lemma $1, a \in\left(\Delta_{r}^{-1} v^{1 / p}\right)^{-1} \cdot c s$ implies that

$$
R \in\left(\Delta_{r}^{-1} v^{1 / p}\right)^{-1} \cdot c_{0}
$$

and consequently

$$
R_{n} \sum_{k=1}^{n-1} \Delta x_{k} \rightarrow 0 \quad(n \rightarrow \infty)
$$

From $a \in c s,(2.4),(2.6)$ and (2.8), we conclude that

$$
\sum_{k=1}^{\infty} a_{k} x_{k}=-\sum_{k=1}^{\infty} R_{k} \Delta x_{k}+x_{1} \sum_{k=1}^{\infty} a_{k}
$$

and $a x \in c s$. Thus $a \in\left[S_{r}(p, \Delta)\right]^{\beta}$. Now, let $a \in\left[S_{r}(p, \Delta)\right]^{\beta}$. Then $a x \in c s$ for all $x \in S_{r}(p, \Delta)$ and $e \in S_{r}(p, \Delta)$. This implies that $a \in c s$. Let $v \in c_{0}^{+}$be given. Then $x=\Delta_{r}^{-1} v^{1 / p} \in S_{r}(p, \Delta)$. Hence $a \in\left(\Delta_{r}^{-1} v^{1 / p}\right)^{-1} \cdot c s$, and by Lemma 1, we get (2.7). Therefore (2.8) holds for all $x \in S_{r}(p, \Delta)$. By (2.4), we get $R \Delta x \in c s$. Since $x \in S_{r}(p, \Delta)$ if and only if $y=\Delta_{r} x=\left(k^{r} \Delta x_{k}\right)_{k=1}^{\infty} \in c_{0}(p)$, this implies that

$$
\sum_{k=1}^{\infty}\left|R_{k}\right| \frac{N^{-1 / p_{k}}}{k^{r}}<\infty
$$

for some integer $N \geq 2$ (cf. [9], Theorem 6). Hence $\left[S_{r}(p, \Delta)\right]^{\beta}=C_{r}(p)$.

\section{MATRIX TRANSFORMATIONS}

For any infinite complex matrix $A=\left(a_{n k}\right)_{n, k=1}^{\infty}$, we write $A_{n}=\left(a_{n k}\right)_{k=1}^{\infty}$ for the sequence in the $n$th row of $A$. Let $X$ and $Y$ be two subsets of $w$. By $(X, Y)$, we denote the class of all matrices $A$ such that the series $A_{n}(x)=\sum_{k=1}^{\infty} a_{n k} x_{k}$ converges for all $x \in X$ and each $n \in \mathbb{N}$, and the sequence $A x=\left(A_{n}(x)\right)_{n=1}^{\infty} \in Y$ for all $x \in X$.

THEOREM 3.1. Let $p=\left(p_{k}\right)_{1}^{\infty}$ be a strictly positive sequence and $r \geq 1$. Then $A \in\left(S_{r}(p, \Delta), \ell_{\infty}\right)$ if and only if

(i)

$$
\begin{aligned}
D_{r}(v): & =\sup _{n}\left|A_{n}\left(\Delta_{r}^{-1} v^{1 / p}\right)\right| \\
& =\sup _{n}\left|\sum_{k=1}^{\infty} a_{n k} \sum_{j=1}^{k-1} \frac{v^{1 / p}}{j^{r}}\right|<\infty \quad \text { for all } v \in c_{0}^{+},
\end{aligned}
$$

(ii)

$$
D_{r}(M):=\sup _{n}\left(\sum_{k=1}^{\infty}\left|R_{n k}\right| \frac{M^{1 / p_{k}}}{k^{r}}\right)<\infty \text { for some integer } \quad M \geq 2,
$$

where $R_{n k}=\sum_{j=k+1}^{\infty} a_{n k}$ for all $n$ and $k$, and

$$
D_{\infty}:=\sup _{n}\left|A_{n}(e)\right|=\sup _{n}\left|\sum_{k=1}^{\infty} a_{n k}\right|<\infty .
$$


PROOF. Let the conditions (i), (ii) and (iii) be true and $x \in S_{r}(p, \Delta)$. By Theorem 2.1(b), conditions (i) and (ii) imply that $A_{n} \in\left[S_{r}(p, \Delta)\right]^{\beta}$ for $n=1,2, \ldots$ for a given $M \in \mathbb{N}$, there exists a $M^{\prime}=M^{\prime}(M) \in \mathbb{N}$ such that $\sup _{k \geq M^{\prime}}\left|k r \Delta x_{k}\right| \leq \frac{1}{M}$, where $M \geq 2$ is the integer in (ii). By (2.9), we have

$$
\left|A_{n}(x)\right| \leq D_{r}(M)+\left|x_{1}\right| D_{\infty} \quad(n=1,2, \ldots)
$$

and hence $A x \in \ell_{\infty}$. Conversely, let $A \in\left(S_{r}(p, \Delta), \ell_{\infty}\right)$. Since $x=\Delta_{r}^{-1} v^{1 / p} \in S_{r}(p, \Delta)$ for all $v \in c_{0}^{+}$, condition (i) follows immediately. Also the necessity of (iii) follows from the fact that $x=e \in S_{r}(p, \Delta)$. Now, by (i), (iii) and (2.9),

$$
\mathrm{A}_{n}(x)=-\sum_{k=1}^{\infty} R_{n k} \Delta x_{k}+x_{1} A_{n}(e) \quad(n=1,2, \ldots) .
$$

Since $A x \in \ell_{\infty}$ and $x_{1} A e \in \ell_{\infty}$, therefore $\left(R_{n} \Delta x\right)_{n=1}^{\infty} \in \ell_{\infty}$. Since $x \in S_{r}(p, \Delta)$ if and only if $\left(k^{r} \Delta x_{k}\right)_{k=1}^{\infty} \in c_{0}(p)$, and $\left(\sum_{k=1}^{\infty}\left(R_{n k} / k^{r}\right)\left(k^{r} \Delta x_{k}\right)\right)_{n=1}^{\infty} \in \ell_{\infty}$ for all $\left(k^{r} \Delta x_{k}\right)_{k=1}^{\infty} \in c_{0}(p)$, this implies that $D_{r}(M)<\infty$ for some integer $M \geq 2$, and (ii) holds.

THEOREM 3.2. Let $p=\left(p_{k}\right)_{1}^{\infty}$ be a strictly positive sequence such that $p \in \ell_{\infty}$, and $r \geq 1$. Then $A \in\left(S_{r}(p, \Delta), \ell_{1}\right)$ if and only if

$$
\begin{aligned}
C_{r}^{(1)}(v): & =\sup _{\substack{N \subseteq \mathbb{N} \\
N \text { finite }}}\left|\sum_{n \in N} A_{n}\left(\Delta_{r}^{-1} v^{1 / p}\right)\right| \\
& =\sup _{\substack{N \subseteq \mathbb{N} \\
N \text { finite }}}\left|\sum_{n \in N} \sum_{k=1}^{\infty} a_{n k} \sum_{j=1}^{k-1} \frac{v^{1 / p},}{j^{r}}\right|<\infty
\end{aligned}
$$

for all sequences $v \in c_{0}^{+}$,

$$
\mathrm{C}_{r}^{(2)}(M):=\sup _{\substack{N \in \mathbb{N} \\ N \text { finit }}}\left(\sum_{k=1}^{\infty} \sum_{n \in N}\left|R_{n k}\right| \frac{M^{-1 / p_{k}}}{k^{r}}\right)<\infty
$$

for some integer $M \geq 2$, and

$$
D_{r}^{(3)}:=\sup _{\substack{N \subset \mathbb{N} \\ N \text { finite }}}\left|\sum_{n \in N} A_{n}(e)\right|<\infty .
$$

PROOF. Let conditions (i), (ii) and (iii) hold. Then $A_{n} \in\left[S_{r}(p, \Delta)\right]^{\beta} \cdot$ Let $x S_{r}(p, \Delta)$. For a given $M \in \mathbb{N}$ there exists a $M^{\prime}=M^{\prime}(M) \in \mathbb{N}$ such that $\sup _{k \geq M^{\prime}}\left|k^{r} \Delta x_{k}\right|^{p_{k}}<\frac{1}{M}$. Now, by (2.9) and the inequality in [10], p. 33, we have

$$
\sum_{n=1}^{m}\left|A_{n}(x)\right| \leq 4\left(C_{r}^{(2)}(M)+\left|x_{1}\right| D_{r}^{(3)}\right)<\infty
$$

Since $m \in N$ is arbitrary, we have $A x \in \ell_{1}$. Conversely, let $A \in\left(S_{r}(p, \Delta), \ell_{1}\right)$. Then

$$
\left|\sum_{n \in N} A_{n}(x)\right| \leq \sum_{k=1}^{\infty}\left|A_{n}(x)\right|<\infty
$$

for all $x \in S_{r}(p, \Delta)$ and for all finite subsets $N$ of $\mathbb{N}$. Therefore the necessity of (iii) and (i) follows immediately, since $e$ and $x=\Delta_{r}^{-1} v^{1 / p} \in S_{r}(p, \Delta)$ for every sequence $v \in c_{0}^{+}$. Further we have 


$$
\left(\sum_{k=1}^{\infty} \frac{R_{n k}}{k^{r}} k^{r} \Delta x_{k}\right)_{n=1}^{\infty} \in \ell_{1} \text { for all }\left(k^{r} \Delta x_{k}\right)_{k=1}^{\infty} \in c_{0}(p),
$$

and hence (ii) holds by Lemma 2 .

ACKNOWLEDGMENT. (*) This research is supported by the University Grant Commission, number F.8-14/94. The authors are grateful to the referee for his or her valuable suggestions which improved the clarity of this presentation.

\section{REFERENCES}

[1] CHOUDHARY, B. and MISHRA, S.K., A note on certain sequence spaces, J. Analysis, 1 (1993), 139-148.

[2] LASCARIDES, C.G., A study of certain sequence spaces and a generalization of a theorem of lyer, Pacific J. Math. 38 (2) (1971), 481-500.

[3] LASCARIDES, C.G. and MADDOX, I.J., Matrix transformations between some classes of sequences, Proc. Cambridge Phil. Soc. 68 (1970), 99-104.

[4] SIMONS, S., The sequence spaces $\ell\left(p_{\nu}\right)$ and $m\left(p_{\nu}\right)$, Proc. London Math. Soc. 15 (1965), $422-$ 436.

' [5] AHMAD, Z.U. and MURSALEEN, Köthe-Toeplitz duals of some new sequence spaces and their matrix maps, Publ. Inst. Math. (Beograd) 42 (56) (1987), 57-61.

[6] KIZMAZ, H., On certain sequence spaces, Canadian Math. Bull. 24 (1981), 169-175.

[7] MALKOWSKY, E., A note on the Köthe-Toeplitz duals of generalized sets of bounded and convergent difference sequences, J. Analysis 3 (1995).

[8] MALKOWSKY, E., MURSALEEN and QAMARUDDIN, Generalized sets of difference sequences, their duals and matrix transformations (unpublished).

[9] MADDOX, I.J., Continuous and Köthe-Teplitz duals of certain sequence spaces, Proc. Camb. Phil. Soc. 65 (1967), 431-435.

[10] PEYERIMHOFF, A., Über ein Lemma von Hern Chow, J. London Math. Soc. 32 (1957), 33-36. 


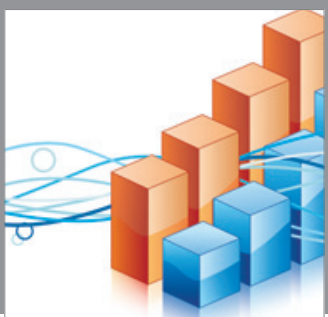

Advances in

Operations Research

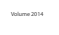

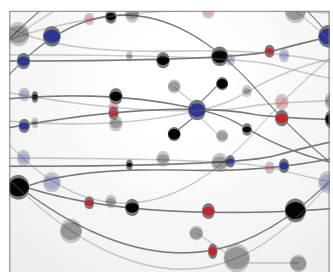

\section{The Scientific} World Journal
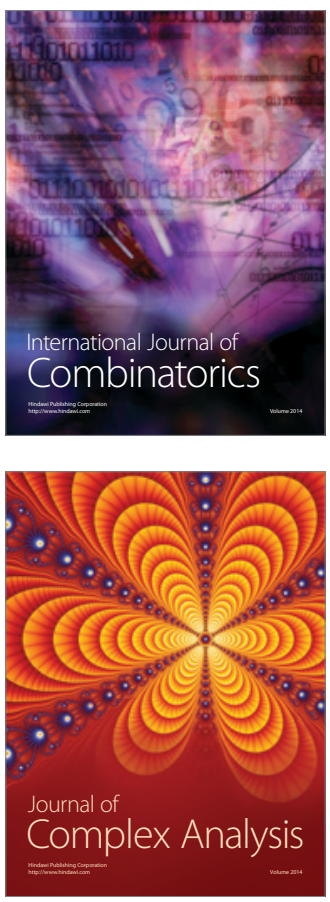

International Journal of

Mathematics and

Mathematical

Sciences
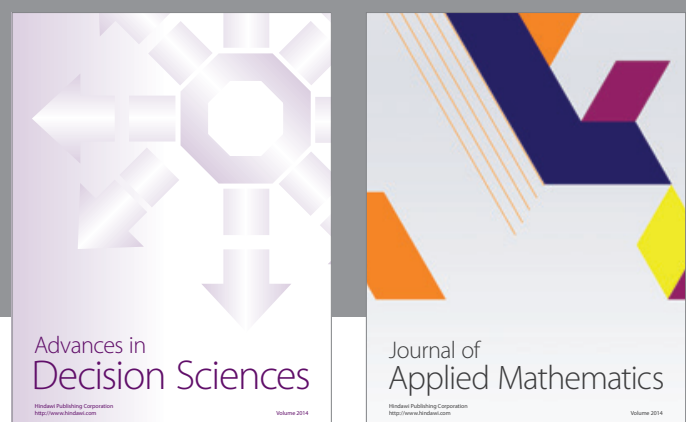

Journal of

Applied Mathematics
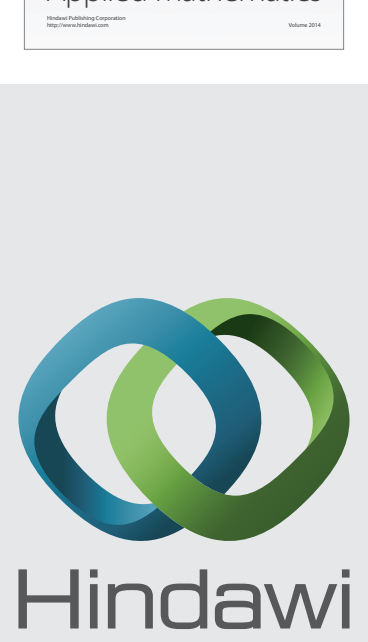

Submit your manuscripts at http://www.hindawi.com
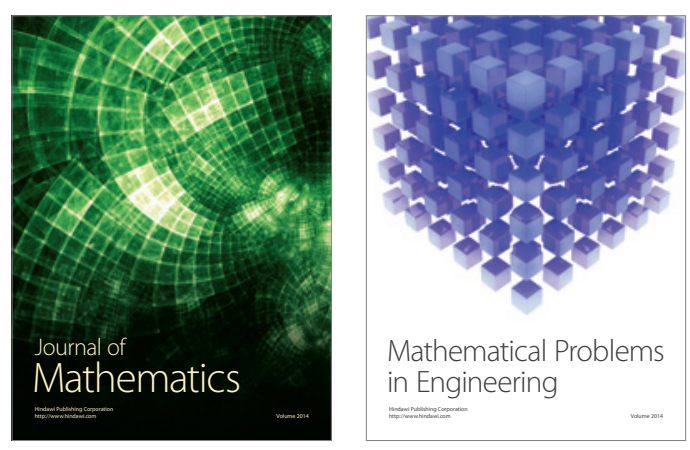

Mathematical Problems in Engineering
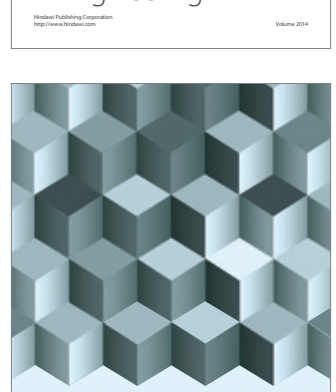

Journal of

Function Spaces
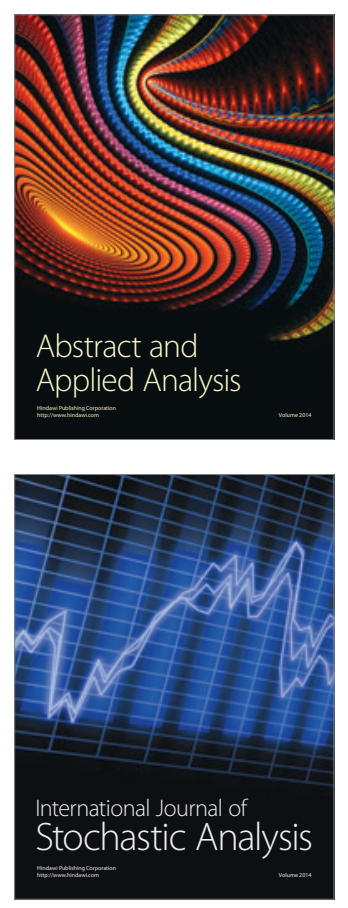

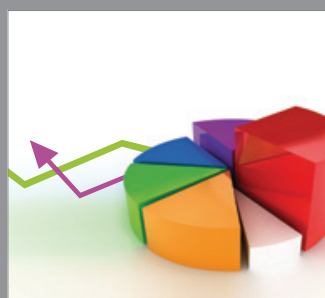

ournal of

Probability and Statistics

Promensencen
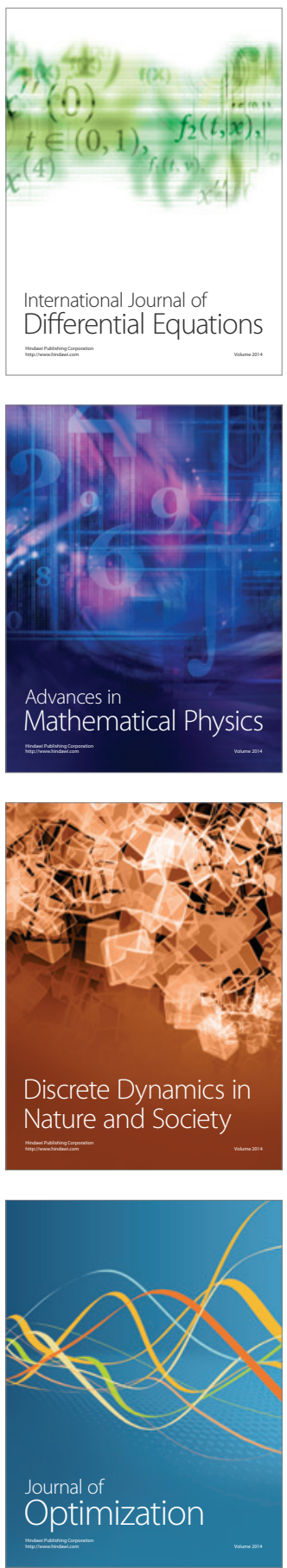